\title{
Cytological Changes of Oral Cavity and of High/Low Risk HPV Detection in Women with Cervical Pathology
}

\author{
Nestan Shonia1,2, Keti Gogilashvili1,2, Irina Mepharishvili1,2, Teona Muzashvili1,2, \\ George Burkadze1,2* \\ ${ }^{1}$ Ilia State University, Tbilisi, Georgia \\ ${ }^{2}$ Tbilisi State Medical University, Tbilisi, Georgia \\ Email: ${ }^{*}$ gburkadze@hotmail.com
}

Received 20 April 2015; accepted 13 June 2015; published 17 June 2015

Copyright (C) 2015 by authors and Scientific Research Publishing Inc.

This work is licensed under the Creative Commons Attribution International License (CC BY). http://creativecommons.org/licenses/by/4.0/

\section{(c) (i) Open Access}

\section{Abstract}

Oropharyngeal tumor is the eight most common cause of cancer death worldwide. Among the causes of oropharyngeal carcinoma significant are cigarette smoking, abuse of alcohol, multipartners, high risk HPV (Human Papillomavirus) and etc. Cytological material (oral Pap smear) was taken from oral fundus and cheek mucous by the cytobrush. 47 participants with cervix pathology and 42 participants of the control group were investigated. Oral smears were stained by the Papanicolaou method and were diagnosed by a double blind method. After the cytological examination, we carried out detecting low and high risk HPV by chromogenic in-situ hybridization (CISH method) to use a positive and negative control. The quantitative statistical analysis was performed by SPSS V.19.0. Numeral data were processed using Pearson correlation and $\mathrm{X}^{2}$ tests. Confidence interval of $95 \%$ was regarded statistically significant. Study group's oral pap smear with atypical cytology was detected in $61.7 \%$. Atypical cytological changes in the control group were confirmed in $\mathbf{3 8 . 0} \%$. High risk HPV detection by the CISH, in study group, showed positivity in $46.8 \%$ and in the control group-in $4.8 \%$. Participants with cervix pathology will be considered as a risk-group to develop oropharyngeal tumor. Screening program for oropharyngeal cancer combines Pap smear and high risk HPV test together. For the control group, it is recommended to use oral pap smear and in the case of atypical changes using high risk HPV test too.

\section{Keywords}

Oropharyngeal Cancer, Oral Cytology, Pap Smear, HPV Test, CISH

\footnotetext{
${ }^{*}$ Corresponding author.
}

How to cite this paper: Shonia, N., Gogilashvili, K., Mepharishvili, I., Muzashvili, T. and Burkadze, G. (2015) Cytological Changes of Oral Cavity and of High/Low Risk HPV Detection in Women with Cervical Pathology. Health, 7, 715-722. 


\section{Introduction}

Oropharyngeal tumor is the eight most common cause of cancer death worldwide.

This year 45,780 cases of oropharyngeal cancer have been diagnosed in the United States. This cancer is probably increasing because of infection with HPV. It is estimated that 8650 deaths from this disease will occur this year. The 5-year survival rate of people with oropharyngeal cancer is $63 \%$ and the 10 years survival rate is $51 \%$. If the cancer is diagnosed at an early stage, the 5-year survival rate is $83 \%$. But when the cancer invasion in adjacent organs and/or regional lymph nodes, the five years' survival rate is $61 \%$. In case of distant metastases in the body this number is 37\% [1]-[3]. This incidence varies widely among different regions. In North America and Europe it consist 3\% - 4\% of all cancer diagnosis. Conversely, in the Southeast Asia and Africa it accounts approximately $8 \%$ - 10\% [4]. Among the reasons that cause oropharyngeal carcinoma are cigarette smoking, abuse of alcohol, multipartners, high risk HPV and etc. In recent study, the role of HPV is confirmed in oropharyngeal tumors [5] [6] [10]. Significantly advance is that oropharyngeal tumors develop from the pre-malignant condition's further progression and we are eligible to detect the disease earlier and prevent further complications using cytological investigation and HPV test [7]-[9].

There are no screening programs for oropharyngeal tumors in Georgia. It is important in many developing countries including Georgia, because they have a huge number of risk factors of further tumor development such as cigarette smoking, abuse of alcohol, low hygiene of oral cavity, chronic pathologies of teeth, gums etc. It is known that women with cervical displastic lesions, already represent infection caused by high risk HPV. We aimed to investigate oral cavity smear, cytologically, in women with dysplastic changes of cervix, with subsequent HPV testing.

\section{Material and Methods}

We investigated 53 participants with cervix pathology and 46 participants of the control group. The participants from the control group did not belong to risk-group of oripharyngeal tumors and did not have cervix pathology. Age range was 23 - 68 years $(n=99)$. Participants were devided into 3 groups using of 15 years interval for detection of age peculiarities. I group: 23 - 38 years (cervix pathology $n=15$, control $n=12$ ), II group: $39-53$ years (cervix pathology $n=24$, control group, $n=23$ ), III group: 54 - 68 years (cervix pathology $n=8$, control group $n=7$ ). Cytological material (oral Pap smear) was taken from oral fundus and cheek mucous by the cytobrush. Oral smears were stained using the Papanicolaou technique and were diagnosed by a double blind method. Firstly, in the cytological material squamous cell adequacy was assessed, which included superfacial, intermediate and parabasal cells existence. Oral smear, without of parabasal cells, was assessed as inadequate. There were not seen koilocytes, damaged squamous cells were assessed according to cell enlargement, polymorphism, irregularity of nuclear membrane, nuclear-cytoplasmic index, number of nuclei, binuclei, keratinization, hyperchromatism and chromatin distribution. For assessment of cytological material the study group was classified in 5 basic groups: inadequate, NILM (Negative for intraepithelial lesion and malignancy), ASCUS (atypical squamous cells undetermined significance), SIL (squamous intraepithelial lesion) and cancer. After the cytological diagnosis we detect and identify low and high risk HPV by chromogenic in-situ hybridization (CISH method) to use a positive and negative control. The positive results were assessed to use of light microscope with exist of the red nuclei. For the detection of low risk HPV subtypes was used HPV probe: HPV type 6/11 Probe and chromogenic visualization system: Zyto Fast PLUS CISH Implementation Kit AP-NBT/BCIP. For the detection of high risk HPV subtypes was used HPV probe: HPV type 16/18/31/35/45/51/82 Probe and chromogenic visualization system: Zyto Fast PLUS CISH Implementation Kit AP-NBT/BCIP.

The quantitative statistical analysis was performed by SPSS V.19.0. Numeral data were processed using Pearson correlation and $\mathrm{X}^{2}$ tests. Confidence interval of $95 \%$ was regarded statistically significant.

\section{Results}

The study of oral cavity cytology in women with cervix pathology showed that out of 53 participants 6 (11.3\%) turned out to have inadequate oral cavity cytology.

Adequate smear were detected in 47 (88.7\%), out of which NILM was detected in 18 (38.2\%). In 19 (40.4\%) were detected ASCUSand in 10 (21.3\%)-SIL. Altogether, cytology of oral cavity with atypical changes was detected in 27 (61.7\%). Squamous cell carcinoma was not detected at all (Tables 1-4). 
Table 1. Oral Pap smear, study group and control group—-distribution of cytology and HPVPap/HPV CISH(+) N = 47 - 42.

\begin{tabular}{cccc}
\hline \multirow{2}{*}{ Group under investigation } & NILM & ASCUS & SIL \\
\cline { 2 - 4 } & Study group control—HPV & Study group control—HPV & Study group control—HPV \\
\hline I group 23 - 38 years & $40 \%-6 / 13.3 \%-2$ & $33.3 \%-5 / 20.0 \%-3$ & $26.7 \%-4 / 20.0 \%-3$ \\
$\mathrm{n}=15-12$ & $58.3 \%-7 / 0 \%-0$ & $33.3 \%-4 / 8.3 \%-1$ & $8.3 \%-1 / 0 \%-0$ \\
\hline
\end{tabular}

Table 2. Oral Pap smear, study group and control group—distribution in II group.

\begin{tabular}{cccc}
\hline \multirow{2}{*}{ Group under investigation } & NILM & ASCUS & SIL \\
\cline { 2 - 4 } & Study group control-HPV & Study group control-HPV & Study group control-HPV \\
\hline II group $39-53$ years & $37.5 \%-9 / 12.5 \%-3$ & $41.6 \%-10 / 25.0 \%-6$ & $20.8 \%-5 / 12.5 \%-3$ \\
(n=24 - 23) & $65.2 \%-15 / 0 \%-0$ & $26.1 \%-6 / 4.3 \%-1$ & $8.7 \%-2 / 0 \%-0$ \\
\hline
\end{tabular}

Table 3. Distribution in III group.

\begin{tabular}{cccc}
\hline \multirow{2}{*}{ Group under investigation } & NILM & ASCUS & SIL \\
\cline { 2 - 4 } & Study group control—HPV & Study group control—HPV & Study group control—HPV \\
\hline III group $54-68$ years & $37.5 \%-3 / 0 \%-0$ & $50 \%-4 / 25 \%-2$ & $12.5 \%-1 / 0 \%-0$ \\
(n=8- $)$ & $57.1 \%-4 / 0 \%-0$ & $28.6 \%-2 / 0 \%-0$ & $14.3 \%-1 / 0 \%-0$ \\
\hline
\end{tabular}

Table 4. Total numbers.

\begin{tabular}{cccc}
\multirow{2}{*}{ Group under investigation } & NILM & ASCUS & SIL \\
\cline { 2 - 4 } & Study group control—HPV & Study group control—HPV & Study group control—HPV \\
\hline \multirow{2}{*}{ Total 23 - 68 years $(\mathrm{n}=47$ - 42) copy } & $38.2 \%-18 / 10.6 \%-5$ & $40.4 \%-19 / 23.4 \%-9$ & $21.3 \%-10 / 12.8 \%-5$ \\
& $56.5 \%-26 / 0 \%-0$ & $28.6 \%-13 / 4.8 \%-2$ & $9.5 \%-4 / 0 \%-0$ \\
\hline
\end{tabular}

HPV—Human papillomavirus; CISH—Chromogenic in-situ hybridization; NILM—Negative for intraepithelial lesion and malignancy; ASCUS— Atypical squamous cells undetermined significance; SIL—Squamous intraepithelial lesion.

In the control group were investigated $n=46$, out of which in $4(8.7 \%)$ oral cavity smear was inadequate. Out of adequate cases $(n=42)$, NILM was detected in $26(56.5 \%)$, ASCUSin $12(28.6 \%)$, SIL in 49.5\%. Altogether in 16 (38.0\%) having atypiccytologycal changes. Squamous cell carcinoma was not detected at all.

According to the study age groups in I group (23 - 38 years) was detected $n=15$, out of which NILMin oral cavity was revealed in $6(40.0 \%)$, ASCUS in 5 (33.3\%), SIL in 4 (26.7\%). Altogether atypical lesion was detected in 9 (60.0\%). In II group (39 - 53 years) was detected $n=24$, out of which NILM was revealed in 9 (37.5\%); ASCUS in 10 (41.6\%), SILin 5 (20.8\%). Altogether atypical changes was seen in 15 (62.5\%). In III group (54 - 68 years) was detected $n=8$, out of which NILM was detected in 3 (37.5\%); ASCUS in 4 (50.0\%); SIL in 1 (12.5\%). Altogether atypical lesion was detected in 5 (62.5\%) Figure 1.

According to the control age groups, in I group (23 - 38 years) was detected $n=12$ with NILM 7 (58.3\%), with ASCUS 4 (33.3\%), SIL 1 (8.3\%). Altogether atypical cytological changes were seen in 5 (41.7\%). In II group (39 - 53 years) was detected $n=23$, out of which NILM was detected in 15 (65.2\%), with ASCUS in 6 (26.1\%), SIL in 2 (8.7\%). The whole ofatypical cytological lesions were founded in 8 (34.8\%). In III group (54 68 years) was detected $n=7$, out of which with NILM 4 (57.1\%), with ASCUS 2 (28.6\%), with SIL 1 (14.3\%). Altogether atypical cytological changes were seen in 3 (42.9\%) (Figures 2-4).

The detection of low and high risk HPV by chromogenic in situ hybridization technology showed that low risk HPV were detected neither in a group with cervix pathology nor in the control group. High risk HPV, in study group, was revealed in 5 (10.6\%) of NILM, with ASCUS in 11 (23.4\%) and SIL in 6 (12.8\%) in the oral smear Figure 5. Altogether high risk HPV was detected in 17 (36.2\%) of atypical cytology changes. In the control high risk HPV neither in NILM nor in SIL were detected. High risk HPV were founded in 2 (4.8\%) of ASCUS.

According to the age in study group, in I group (23 - 38 years) high risk HPV was detected in 2 (13.3\%) of those having a NILM, in 3 (20\%) with ASCUS and with SIL in 3 (20\%). Altogether atypical cytology were seen 


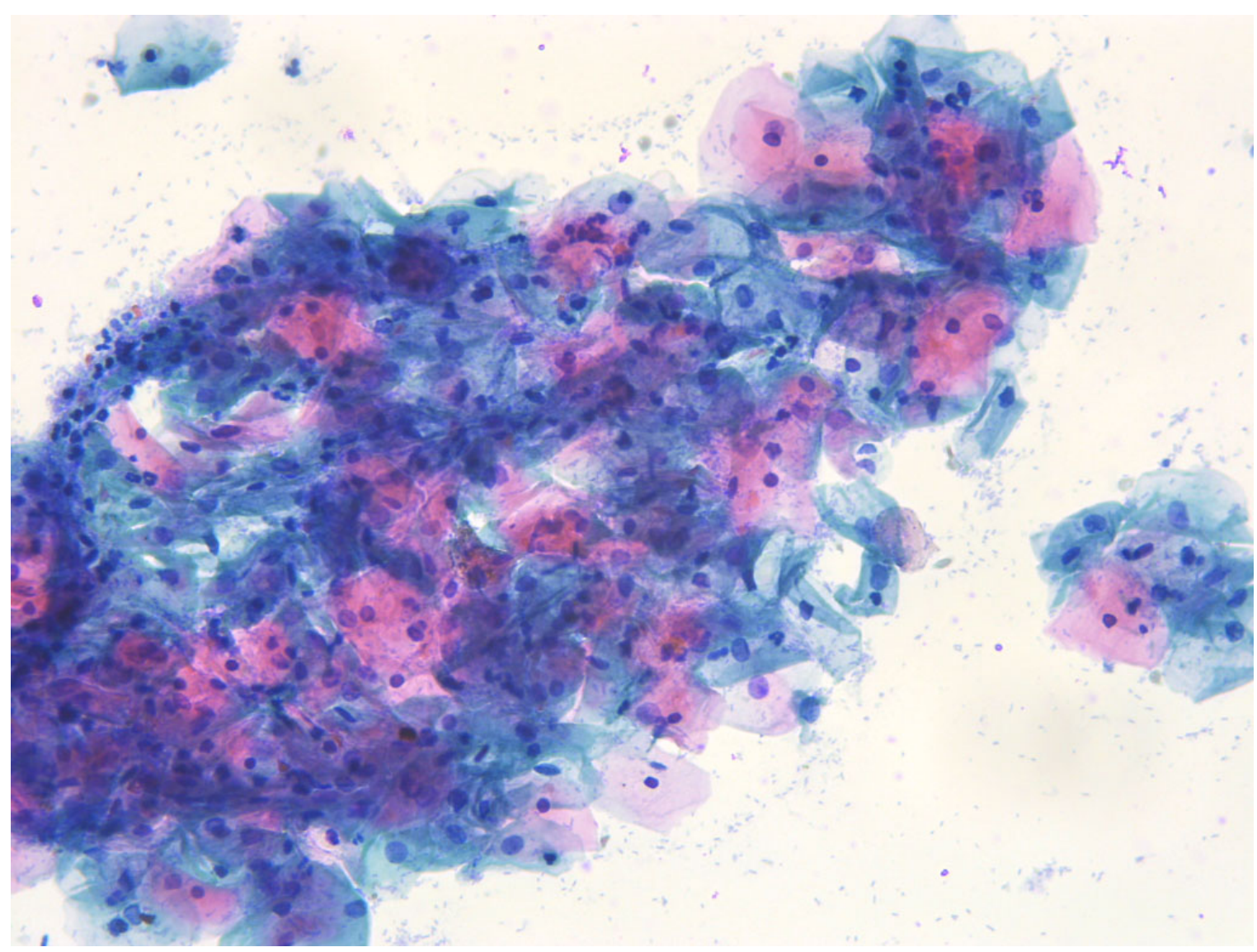

Figure 1. Oral Papsmear-Intermediate cells with an enlarged nucleus and a homogenous chromatin-ASCUS (atypical squamous cells undetermined significance) $40 \times$.

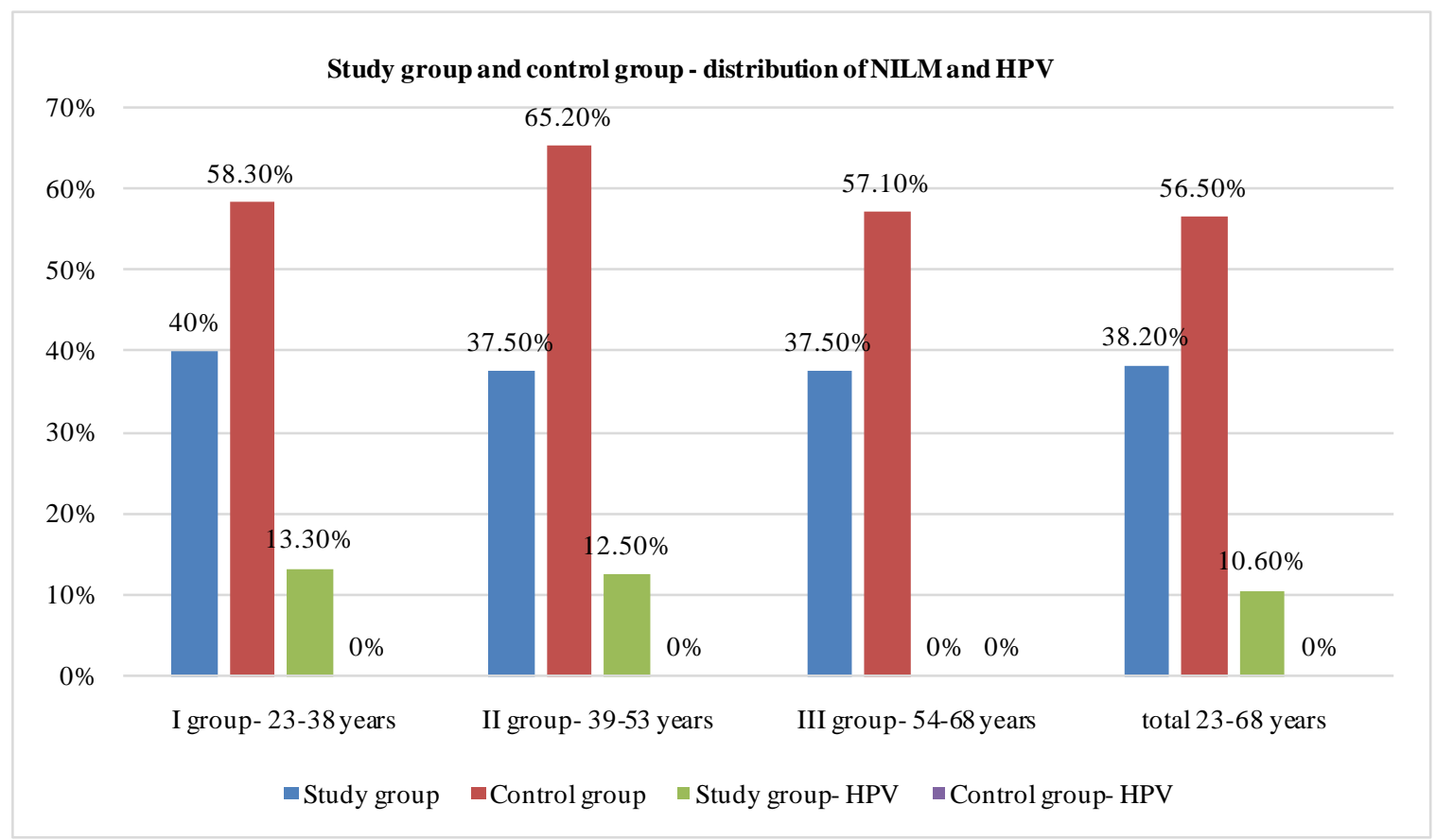

Figure 2. Study group and control group-distribution NILM and HPV. 


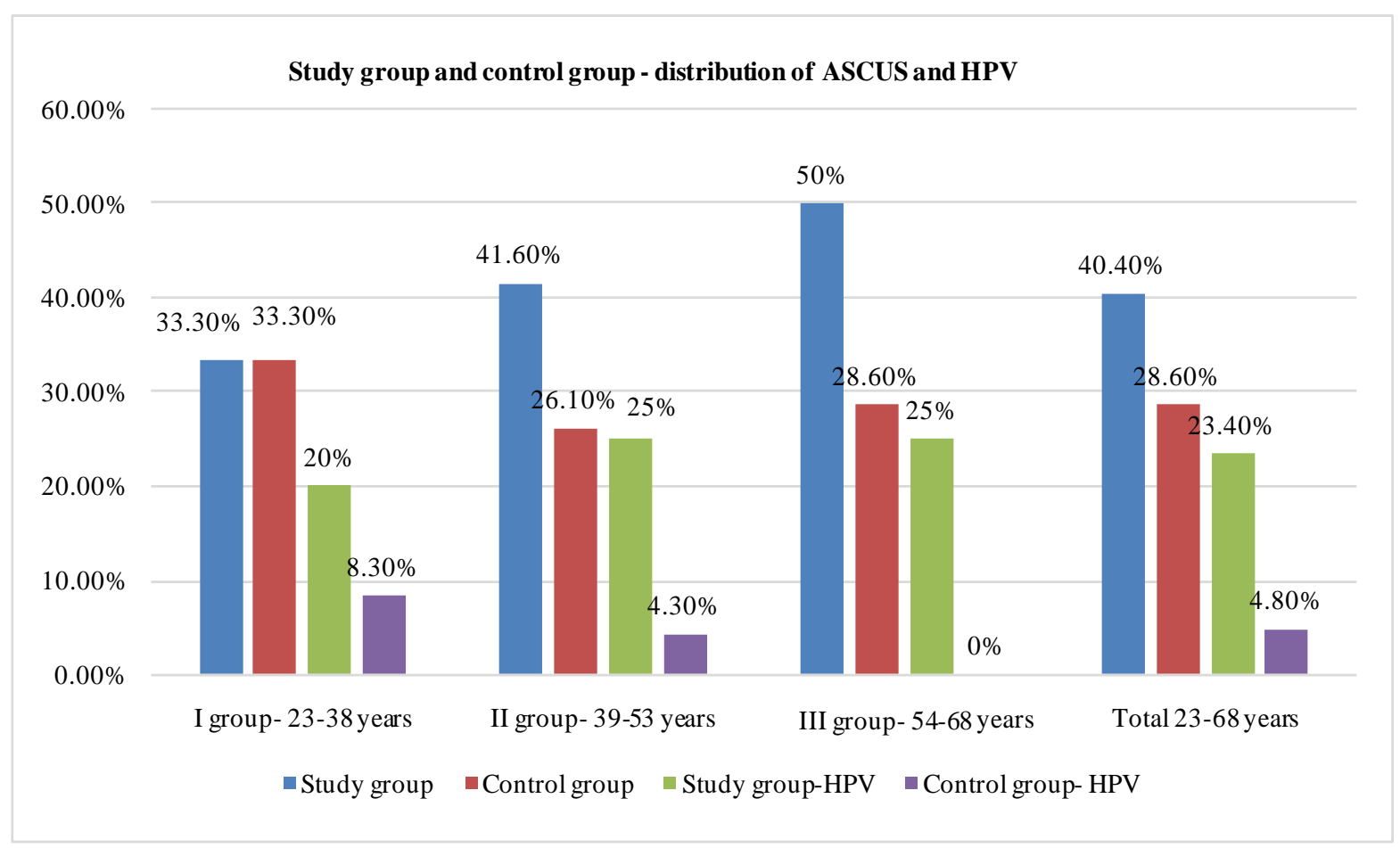

Figure 3. Study group and control group—-distribution of ASCUSand HPV.

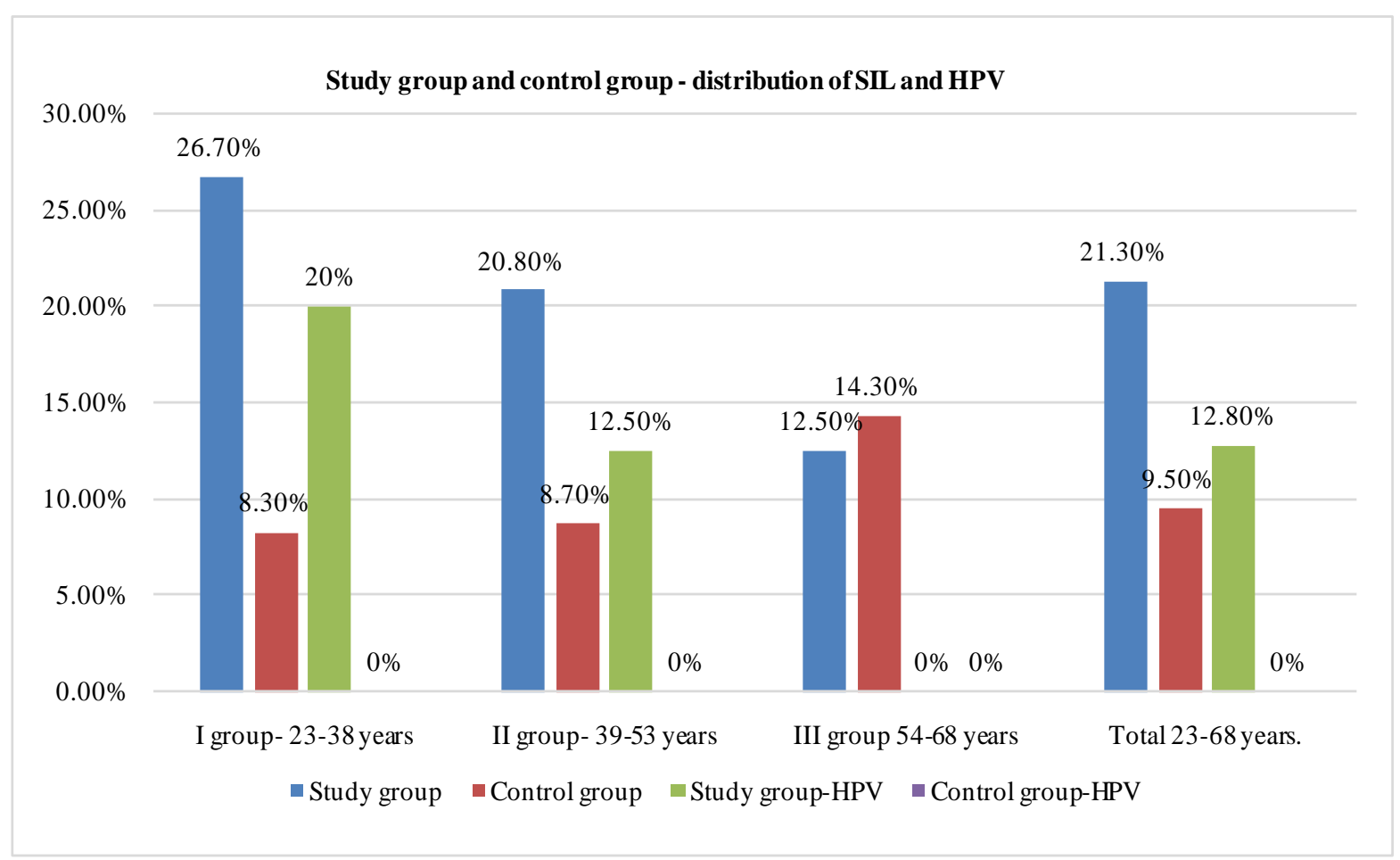

Figure 4. Study group and control group-distribution of SIL and HPV.

in 6 (40\%). In II group (39 - 53 years) with NILM in 3 (12.5\%), with ASCUS in 6 (25\%), with SIL in 3 (12.5\%). Altogether in atypical changes high risk HPV was detected in 9 (60.0\%). In III group (54 - 68 years) in cases of NILM and SIL a high risk HPV was not detected. High risk HPV was detected in 2 (25\%) of ASCUS. 


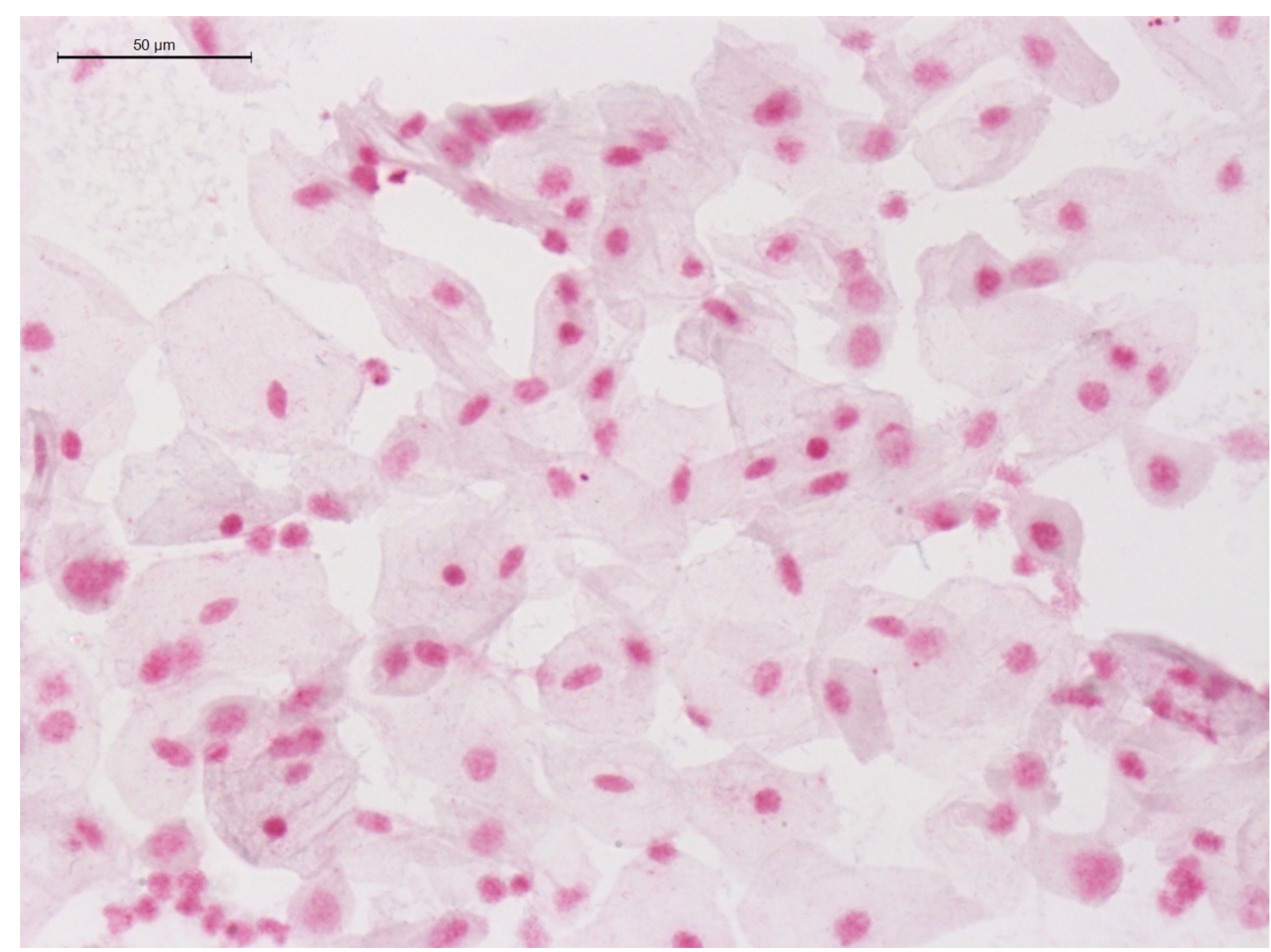

Figure 5. Oral smear-squamous cell nuclei with positive signals on the high risk HPV probe. CISH (in situ hybridization) $40 \times$.

According to the control age groups in I group (23 - 38 years) a high risk HPV is detected in 1 (8.3\%) of ASCUS. In II group (39 - 53 years) in 1 (4.3\%) of ASCUS is detected a high risk HPV. In III group (54 - 68 years) in cases with ASCUS a high risk HPV is not detected.

\section{Discussion}

With our research we want to show that even in case of intact oral cavity (control group) displastic changes and HPV existence are marked and that will be a prerequisite to develop screening program in Georgia. The purpose of a screening test is identify individuals with precursor lesion or early stage cancer more amenable to treatment. Although HPV infection and abnormal cytology were strongly associated with oropharyngealab normalities [11]. And in case of pathology, is carried out cervical screening program for women having cervix pathology. In case of pathology an adequate management is carried out for them. In women having this lesion there are no assess oral cytological changes and accordingly, they are not protected from oral cancer.

Comparative analysis of the oral cavity of participants having cervix pathology and control group showed that cases with NILMin women are 1.5 times less than in the control group. With ASCUS there is 1.4 times more and SIL there is 2.2 times more comparison to the control group. Altogether cases of atypical cytology are 1.6 times more than in the control group. It is famous that, cytopathological abnormality detected in the study population was ASCUS [11]. High risk HPV is detected only in $4.8 \%$ of the control group and only with ASCUS, while high-risk HPV is detected in women with all type of cervix pathology and makes up $46.8 \%$ i.e. It is detected 9.8 times more often than in the control group. Several papers showed that HPV may be involved in the pathogenesis of oral and cervical cancer leading to distinct molecular characteristics compared with HPV negative ones [6]. In the study group the intensity of epithelial lesion directly correlates to detect high risk HPV in 
oral smears. The more is the quality of epithelial lesion the higher is the amount of HPV positive cases. The overall rise in oropharyngeal cancer incidence from 2004 to present is lergely explained by the increasing incidence of HPV positive cancers, wheares incidence of HPV-negative cancers declined [12]. These results strongly support the hupothesis that HPV infection is the cause of rising oropharyngeal canver incidence [13]. High risk HPV is also detected in some case of NILM. In this kind of cases it is possible to detect HPV infection in earlier stages when there are no any reflective cellular changes.

\section{Conclusion}

Participants with cervix pathology will be considered as a risk-group to develop oropharyngeal tumor. The screening program for oropharyngeal cancer combined Pap smear and high risk HPV test together. In the control group, it is recommended to use an oral Pap smear and in case of atypical changes to use a high risk HPV test too.

\section{Acknowledgements}

Authorship contributions: NSH, TM: titerature search; NSH, TM, GB: manuscript writing; IM, GB: study design; NSH, TM, IM: date collection; NSH, KG: date analysis; KG, GB: date interpretation; NSH, TM statistical analysis and figures; NSH, TM, GB: final approval.

\section{Conflict of Interest Statement}

The authors stated that there are no conflicts of interest regarding the publication of this article.

\section{References}

[1] Oral and Oropharyngeal Cancer: Statistics. http://www.cancer.net/cancer-types/oral-and-oropharyngeal-cancer/statistics

[2] Goldenberg, D., Begum, S., Westra, W.H., Khan, Z., Sciubba, J., Pai, S.I., Califano, J.A., Tufano, R.P. and Koch, W.M. (2008) Lymp Node Metastasis in Patient with Head and Neck Cancer: An HPV-Associated Phenomen. Head and Neck, 30, 898-903. http://www.ncbi.nlm.nih.gov/pubmed/18383529 http://dx.doi.org/10.1002/hed.20796

[3] Chaudhary, A.K., Singh, M., Sundaram, S. and Mehrotra, R. (2009) Role of Human Papillomavirus and Its Detection in Potentially Malignant and Malignant Head and Neck Lesions: Updated Review. Head Neck Oncology, 1, 22. http://www.headandneckoncology.org/content/1/1/22 http://dx.doi.org/10.1186/1758-3284-1-22

[4] Santarelli, A., Lo Russo, L., Bambini, F., Campisi, G. and Lo Muzio, L. (2009) New Perspectives in Medical Approach to Therapy of Head and Neck Squamous Cell Carcinoma. Minerva Stomatologica, 58, 445-452. http://www.researchgate.net/publication/38070901_New_perspectives_in_medical_approach_to_therapy_of_head_and neck_squamous_cell_carcinoma

[5] Strurgis, A.K. and Cinciripini, P.M. (2007) Trends in Head and Neck Cancer Incidence in Relation to Smoking Prevalence: An Emerging Epidemic of Human Papillomavirus-Associated Cancers. Cancer, 110, 1429-1435.

[6] Marur, S., D’Souza, G., Westra, W.H. and Forastiere, A.A. (2010) HPV-Associated Head and Neck Cancer: A VirusRelated Cancer Epidemic. Lancet Oncology, 11, 781-789. http://www.ncbi.nlm.nih.gov/pubmed/20451455 http://dx.doi.org/10.1016/S1470-2045(10)70017-6

[7] Kujan, O., Glenny, A.M., Duxbury, J., Thakker, N. and Sloan, P. (2005) Evaluation of Screening Strategies for Improving Oral Cancer Mortality: A Cochrane Systematic Review. Journal of Dental Education, 69, 225-265.

[8] Alfred, B., Christoph, S., Nikolas St. Christian, N., Rita, D. and Norbert, R. (2011) Role of Brush Biopsy and DNA Cytometry for Prevention, Diagnosis, Therapy, and Follow-Up Care of Oral Cancer. Article ID: 875959. http://www.hindawi.com/journals/jo/2011/875959/

[9] Krane, J.F. (2013) Role of Cytology in the Diagnosis and Management of HPV Associated Head and Neck Carcinoma. 57, 117-126. http://www.karger.com/Article/FullText/346715

[10] Pavanello, M.B., Prado, F.B., Balducci, I. and Brandao, A.A. (2006) Cytologic Analysis of Alteration Indiced by Smoking and Alcohol Consumption. Acta Cytologica, 50, 435-440. http://dx.doi.org/10.1159/000325988

[11] Carole, F., Barbara, T. and Douglas, P. (2011) Assosiations between Hpv Infections and Cytopathology: Evaluation of an Oropharyngeal Pap Test in High Risk Populations. http://www.ncbi.nlm.nih.gov/pmc/articles/PMC3380432/ 
[12] Anil, K. Eric, A. and Brenda, M. (2011) Human Papillomavirus and Raising Oropharyngela Cancer Incidence in the United States.

[13] Mark, W., Alessandra, B. and Paul, K. (2012) Low Etiologic Fraction for High-Risk Human Papillomavirus in Oral Cavity Squamous Cell Carcinomas. Oral Oncology, 89, 489-490. http://archive.is/97IEf

\section{Abbreviations}

NILM-Negative for intraepithelial lesion and malignancy;

ASCUS-Atypical squamous cells undetermined significance;

SIL-Squamous intraepithelial lesion;

CISH-In situ hybridization. 